\title{
Validation of low-calorie sweetener(LCS)-focused food frequency questionnaire for assessment of intakes in a free-living adult population: preliminary results
}

\author{
A. Dunne, M. Mulhern, A. M. Gallagher and C. Logue \\ Nutrition Innovation Centre for Food and Health (NICHE), School of Biomedical Sciences, University of Ulster, \\ Coleraine, Northern Ireland, Coleraine, United Kingdom
}

\section{Abstract}

Both obesity and type 2 diabetes mellitus have been associated with excessive free sugar consumption. Increasingly, LCS are used to replace free sugar to reduce the energy density of the diet, whilst maintaining diet palatability. However, while experimental data suggest a beneficial effect of LCS on weight management and glycaemic control, data from free-living populations are contradictory and primarily use reported intakes of low-calorie sweetened beverages as a marker of overall LCS consumption. Therefore, a dietary assessment tool that reliably assesses overall LCS consumption and is applicable in large-scale epidemiological studies is desirable. This study aimed to validate an LCS-focused food frequency questionnaire (FFQ) for assessing habitual dietary intake of the LCS using the method of triads. Healthy adults were recruited at Ulster University, Coleraine $(n=120)$. A 65-item FFQ and a 4-day food diary (FD) were used to assess dietary intake of LCS with amounts based on maximum permitted levels for foods and beverages as per EU legislation. A novel urinary biomarker approach, developed by this research group, was used to objectively assess consumption of 5 commonly used LCS $^{(1)}$. The method of triads was used to validate the FFQ by evaluating the correlations between the FFQ, FD and the urinary biomarkers (reference method). A total of 110 participants (51 males, 59 females) completed all analyses. Median (IQR) total LCS consumption estimated from the FFQ, FD and urinary biomarker was 116.8 (24.9-303.3) mg/d, 27.8 (0.0-247.3) mg/ $\mathrm{d}$ and $5.0(0.6-34.9) \mathrm{mg} / \mathrm{d}$ respectively. Moderate correlations were observed between the $\mathrm{FD}$ and urinary biomarker $(\mathrm{r}=0.471 ; \mathrm{P}<$ $0.001)$ and between the FFQ and FD $(r=0.471 ; \mathrm{P}<0.001)$. There was a low correlation between the FFQ and urinary biomarker $(\mathrm{r}=$ $0.291 ; \mathrm{P}<0.001)$. Using the method of triads, the overall validity co-efficient for the FFQ was moderate $(0.54)$. These preliminary results indicate potential for this LCS-focused FFQ as a method of assessing LCS consumption in free-living populations. However, as maximum permitted levels of LCS in foods and beverages were used to estimate LCS consumption from the FFQ and FD, they are likely to significantly overestimate actual LCS intakes as compared to the more reliable biomarker approach. Therefore, the FFQ ability to categorise individuals into low-, medium- and high LCS consumers will be tested. Further analysis is ongoing to assess the completeness of urine collections using PABA analysis and will also focus on individual LCS intake.

\section{Conflict of Interest}

There is no conflict of interest

\section{Reference}

1. Logue C et al. (2017) J Agric Food Chem 22, 4516-4525. 